\title{
Ces tempêtes extrêmes que l'histoire ne nous a pas encore dévoilées
}

David GOUTX'1, Franck BARAER ${ }^{2}$, Amélie ROCHE ${ }^{3}$, Gwenaële JAN ${ }^{4}$

\author{
1. Météo-France Île-de-France Centre - 73 avenue de Paris - 94165 Saint-Mandé ; e-mail : david.goutx@meteo.fr \\ 2. Météo-France Ouest, BP 49139 - 35091 Rennes cedex 9 ; e-mail : franck.baraer@meteo.fr \\ 3. Cetmef, Technopôle Brest Iroise - rue Pierre Bouguer - 29280 Plouzané ; e-mail : amelie.roche@developpement-durable.gouv.fr \\ ${ }^{4}$ Shom, 13 rue de Châtellier - 29200 Brest ; e-mail : gwenaele.jan@shom.fr
}

\begin{abstract}
RÉSUMÉ. - Les études d'aléa de submersion marine s'attachent à définir des valeurs extrêmes pour les niveaux marins et les houles et à combiner ces valeurs afin de produire un aléa de référence à la côte. Pourtant, ces phénomènes résultent souvent d'un même événement météorologique générateur : les tempêtes. Météo-France, le Cetmef et le Shom ont uni leurs efforts pour étudier les tempêtes menaçant le littoral breton et déterminer des événements extrêmes, dont on déduirait les paramètres hydrodynamiques pour l'étude des risques de submersion marine.

En combinant analyse de données d'archives et ré-analyses numériques (ERA-Intérim), on documente chaque tempête comme on le ferait d'un cyclone et on en dresse une typologie afin d'identifier, au sein de chaque famille, la ou les tempêtes les plus représentatives, ainsi que le rapport d'intensité entre membres fréquents et rares. Le modèle Arpège permet de reconstituer les tempêtes historiques les plus représentatives, puis, la Prévision d'Ensemble du modèle Arpège (PEARP) fournit des tempêtes fictives plausibles qu'on peut rendre concomitantes avec des conditions défavorables de marée.

Cette méthode, qui permet d'étudier des événements extrêmes plausibles que l'histoire ne nous a pas encore dévoilés, pose le problème de la probabilité des évènements fictifs. Elle est néanmoins innovante et pertinente dans l'appréhension des événements extrêmes permettant de mettre à l'épreuve les stratégies d'adaptation de nos territoires littoraux, comme cela est notamment demandé dans le cadre de l'application de la Directive européenne « Inondation » pour l'événement extrême.
\end{abstract}

Mots-clés : modèle de prévision, ré-analyses, tempêtes historiques, tempêtes fictives plausibles

\section{These extreme storms that history has not revealed yet}

\begin{abstract}
Risk studies of coastline flooding strive to define extreme values for sea level and the surf and then combine them to determine a threat index for the seaboard. However, these phenomenona are often generated by the same type of weather event: the storms. Meteo-France, the Cetmef and the Shom got together to study the storms threatening the coast of Brittany and to determine the extreme events the associated hydrodynamic parameters of which would allow to study the risk of coastline flooding.

By combining archives and re-analysis (ERA-Intérim), each storm is documented; then a typology is implemented to establish within each cluster, which elements are the most representative. The Arpege model enables us to restore the most typical historical storms, then the Ensemble Prevision supplies data on fictitious but plausible storms that can be afterwards combined with unfavourable tide conditions.

This method which enables us to study plausible extreme events not yet revealed to us, creates the problem of the likelihood of these fictitious events. It is nonetheless an innovative technique, relevant for the understanding of extreme events so that the strategies used to adapt our shorelines, as it is demanded by the 2007/60/CE European Directive in the case of extreme events, could be assessed.
\end{abstract}

Key-words: forecast model, re-analysis, historical storms, plausible fictitious storm

\section{INTRODUCTION}

\section{I.1. La tempête : un objet conceptuel complexe difficile à saisir}

Il n'existe pas de définition claire de cet être météorologique qu'on appelle " tempête ». Les définitions existantes rendent plutôt compte d'une phénoménologie (vents mesurés supérieurs à un seuil donné ou ayant causé des dommages, des décès, des perturbations de la vie sociale) ou de leur médiatisation (reportages emphatiques mettant en scène des dommages spectaculaires, etc) (Schoenenwald, 2013). La tempête est difficile à saisir comme singularité. Il est malaisé de la délimiter au sein de vastes mouvements atmosphériques instables tant horizontaux (interaction de centres d'actions dépressionnaires et anticycloniques, rencontre d'un flux perturbé avec un front froid) que verticaux (conjonction avec des anomalies de tropopause) à la jonction desquels elle prend naissance et vigueur. Sa nature barocline la rend invisible sur les images satellites. D'ailleurs, la connaissance des mécanismes de formation et de propagation des tempêtes est encore en construction : les tempêtes de 1999 ont par exemple permis de progresser dans la compréhension de ces phénomènes en découvrant le rôle de l'anomalie de tropopause (Rivière et al., 2010).

Cette définition phénoménologique a longtemps convenu aux gestionnaires du risque, qui ont hiérarchisé les tempêtes en fonction des dommages qu'elles ont provoqués. Mais en 2010, la tempête Xynthia, moins violente que les tempêtes Lothar et Martin (1999), a atterri sur les côtes françaises au moment d'une marée haute de fort coefficient, occasionnant 
des submersions marines qui ont tué 39 personnes en Vendée et en Charente Maritime. Le phénomène météorologique ne peut désormais plus être considéré isolément comme source des dommages constatés à terre, mais comme l'un des facteurs intervenant dans une combinaison hydrodynamique menaçant le littoral : surcote dépressionnaire, vent, houle, marée, niveau marin.

La météorologie marine a apporté un cadre à ces phénomènes, à travers une classification rendant compte à la fois de la vitesse des vents et des états de mer qu'ils engendrent au large : l'échelle Beaufort. Ce faisant, elle rend évident le lien d'interdépendance qui existe entre le vent, la surcote dépressionnaire et la houle, que noue l'entité météorologique nommée «tempête», et qui compromet presque à coup sûr une analyse statistique classique tentant de croiser les quantiles calculés isolément pour chacun de ces facteurs.

Suite au retour d'expérience de la tempête Xynthia, Météo-France a mis en place une vigilance "VaguesSubmersions » intégrée au dispositif de la Vigilance sur les phénomènes météorologiques dangereux. Pour faire progresser ses connaissances en matière de tempêtes génératrices de submersions marines, l'établissement a lancé en 2012 le programme d'études «VIMERS » (Baraer et al., 2013) sur le littoral breton, associant ses efforts avec ceux du Cetmef et du SHOM, avec le soutien financier de la DREAL Bretagne, de la Région Bretagne et du FEDER. Il s'agit de dresser un inventaire des tempêtes ayant menacé le littoral breton depuis le XIX ${ }^{\text {ème }}$ siècle, ainsi qu'une description précise mobilisant tous les champs de l'expertise météorologique (données d'archives, simulations numériques de ré-analyses, expertise humaine), puis d'établir une typologie de ces tempêtes permettant d'en caractériser l'aléa et de déterminer des tempêtes de référence pour la gestion des risques, éventuellement plus fortes que les plus fortes tempêtes historiques.

\section{I.2. L'histoire : à la fois indispensable et insuffisante}

La puissance de conviction des évènements historiques a été largement démontrée en matière de prévention des risques dans le champ des inondations continentales : le paradigme qui énonce que ce qui est arrivé (par le passé) peut arriver de nouveau (dans le futur) est facile à comprendre, et il constitue la clef de voûte de ce que pourrait être une culture du risque de submersions marines. De plus, en décrivant précisément les tempêtes passées, on se donne la capacité de reconnaître dans le présent les signaux avant-coureurs du "retour » d'une tempête passée et de parer à ses conséquences.

Pourtant, l'histoire est aussi un cadre à la fois restreint, qui ne nous donne à connaître que les combinaisons tempétueuses qui ont réellement eu lieu sans nécessairement comporter les pires combinaisons possibles, et restrictif en ce qu'il ne nous permet pas d'appréhender librement l'inédit que constitueraient ces pires combinaisons possibles sans les frapper du sceau disqualifiant de l'exagération ou du catastrophisme.

Après avoir été surpris par la combinaison inhabituelle des facteurs hydrodynamiques lors de Xynthia, avons-nous le droit de nous laisser surprendre par une tempête encore plus forte qui rencontrerait les mêmes conditions hydrodynamiques défavorables? L'histoire ne nous ayant dévoilé qu'une partie seulement des tempêtes possibles, l'objectif du programme d'études VIMERS est de proposer des tempêtes de référence qui soient indifféremment historiques ou fictives, pourvu qu'elles soient plausibles et réalistes, et auxquelles on a peut-être déjà échappé de peu sans le savoir grâce à une conjonction hydrodynamique favorable.
Nous présentons d'abord les efforts de documentation des tempêtes historiques, puis les analyses typologiques permettant de hiérarchiser les tempêtes en fonction de leurs caractéristiques météorologiques (et non des dommages occasionnés), et enfin, la démarche de prospection des pires tempêtes plausibles.

\section{DOCUMENTER LES TEMPETES HISTORIQUES}

\section{II.1. Valorisation d'archives indispensables et méconnues}

Les bases de données météorologiques comportent de nombreuses informations sur les températures, les pressions, les vents et les précipitations, pour la période de 1951 à nos jours. Mais en fait, les phénomènes tempétueux ne peuvent être saisis correctement que dans une approche dynamique à laquelle les simulations de ré-analyse donnent accès. De telles ré-analyses, basées sur les données météorologiques disponibles au format numérique dans les bases de données, existent pour la période courant de 1979 à nos jours (projet ERA-Intérim du Centre Européen de Prévision, CEP) : avec une résolution spatiale de $80 \mathrm{~km}$ au pas de temps de $6 \mathrm{~h}$, elles sont compatibles avec l'échelle spatio-temporelle des tempêtes. D'autres ré-analyses existent sur la période 1951-2010 (projet ERA-40 du CEP) et sur le $X^{\text {ème }}$ siècle (National Oceanic and Atmospheric Administration, NOAA), mais avec des résolutions spatiale et temporelle insuffisantes pour l'analyse des tempêtes. Et surtout, elles n'exploitent pas les données d'archives disponibles dans des formats physiques hétérogènes qui en rendent la valorisation laborieuse.

Météo-France, le Shom et le Cetmef ont lancé conjointement un effort de valorisation de ces archives pour documenter le plus exhaustivement possible les tempêtes historiques (exemples de documents Fig. 1 à 4).

Ainsi ont pu être exploités les précieux BQE (Bulletins Quotidiens d'Etudes) de Météo-France, comportant des informations barométriques et anémométriques, des cartes pointées à l'échelle synoptique adaptée aux phénomènes tempétueux et des expertises météorologiques formulées et commentées par les spécialistes de l'époque. De vieux barogrammes ont aussi été exhumés des archives pour livrer des informations précieuses sur l'évolution continue des pressions durant un évènement tempétueux ancien. Dans certains cas, l'interprétation de barogrammes insuffisamment normalisés a nécessité la recherche d'informations sur la métrologie historique (Fig. 3). Des observations réalisées à bord des navires et retranscrites dans des bulletins ont aussi pu être exploitées, alimentant ainsi un travail minutieux de compilation d'informations rendant aussi précisément compte que possible de la réalité des tempêtes anciennes. Le Cetmef a commencé à récolter les relevés météorologiques auxquels les services des Phares et Balises ont procédé dans les phares en mer, et le Shom a entamé la numérisation des archives météorologiques rattachées à leurs marégraphes dans la zone d'étude, inventoriés lors des travaux de Nicolas Pouvreau (2008).

\section{II.2. Revisiter les évènements historiques supposés connus}

Les archives permettent de relire d'un œil critique les ré-analyses de la NOAA et du CEP et de prendre du recul 


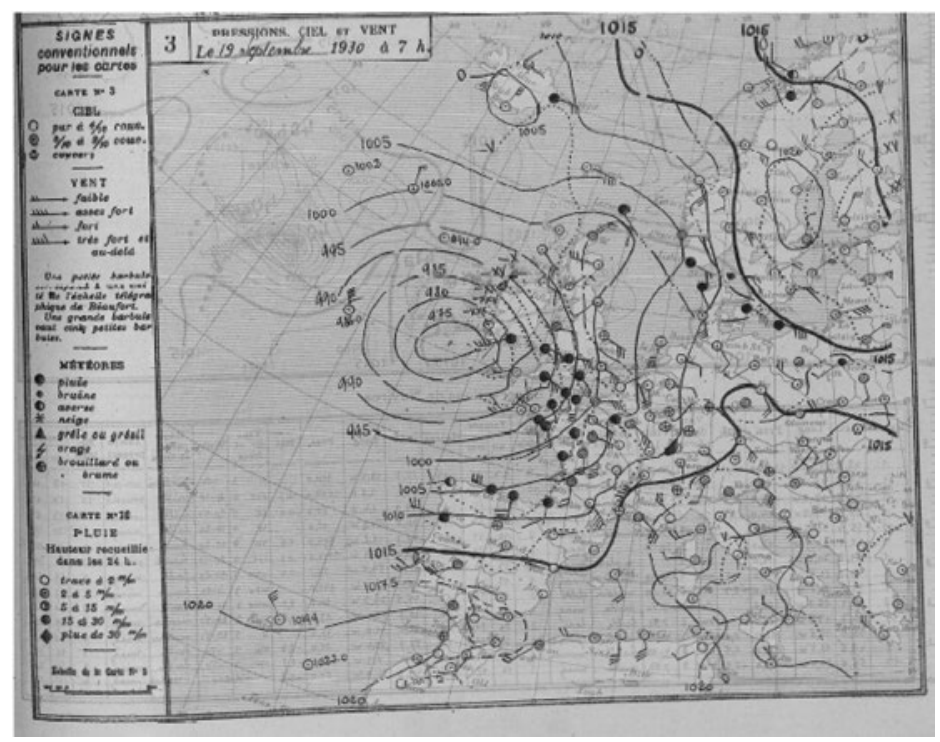

Fig. 1 : Bulletin Quotidien d'Etudes (BQE) du 19 septembre 1930 (Météo-France).

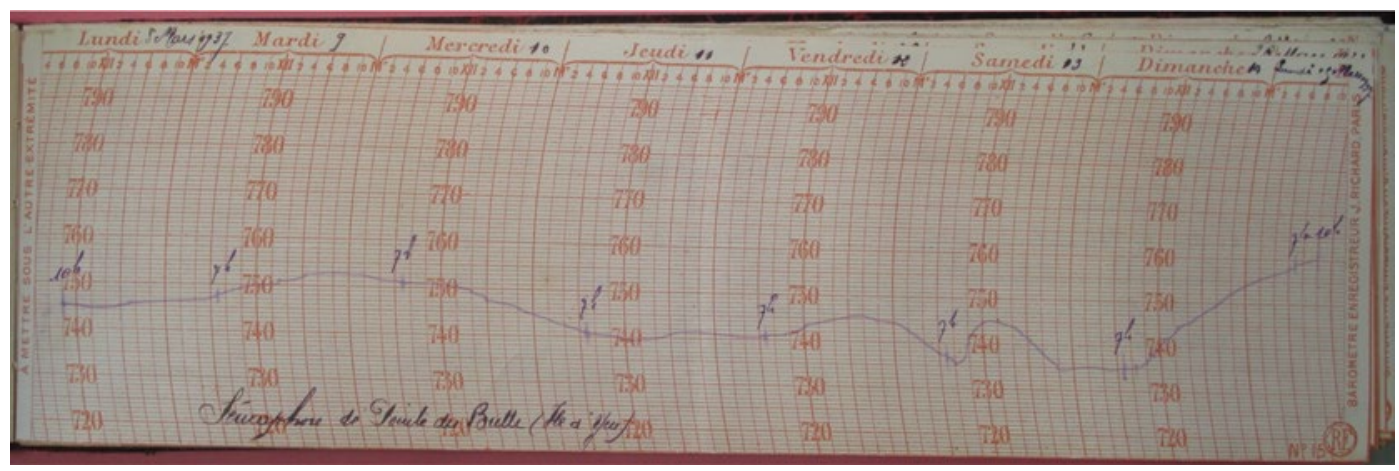

Fig. 2 : Barogramme du sémaphore de l'île d'Yeu du 8 au 14 mars 1937 (Météo-France).

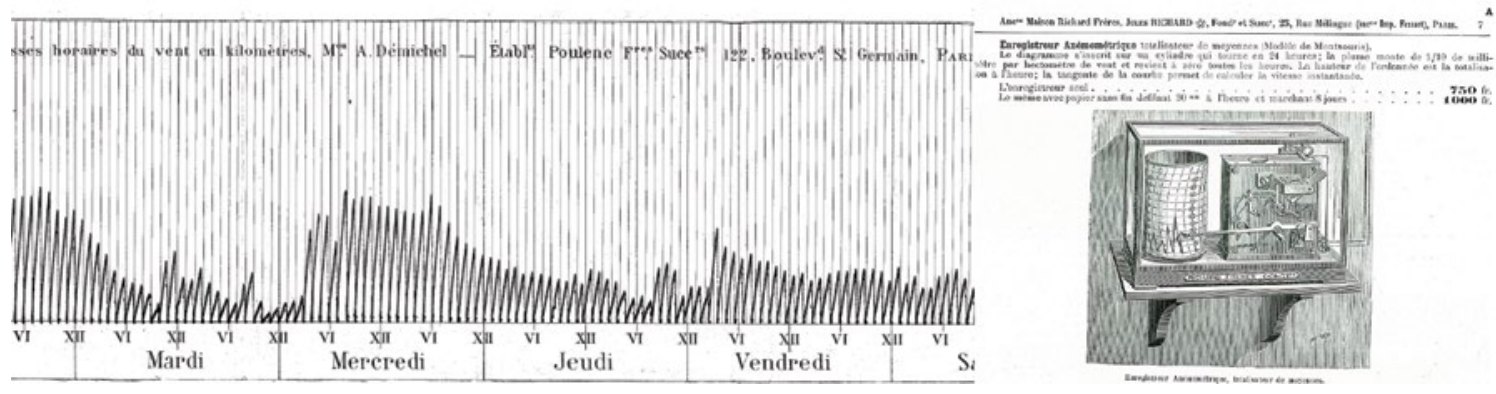

Fig. 3 : Barogramme de 1904 et gravure du barographe figurant l'échelle verticale de déplacement du stylet (Météo-France).

par rapport à l'élégance formelle de ces ré-analyses. Il ne s'agit pas de disqualifier ces précieuses simulations, mais d'enrichir l'ébauche synoptique qu'elles constituent avec les données disponibles et formuler, à dire d'expert, une nouvelle analyse synoptique de la situation tempétueuse.

Pour la tempête de la mi-mars 1937 par exemple, les ré-analyses de la NOAA laissent croire qu'il n'y a eu qu'une seule tempête, creusée à $985 \mathrm{hPa}$ environ et suivant la trajectoire de Xynthia (Garnier, 2012).

Pourtant, lorsqu'on examine le barogramme du sémaphore de l'île d'Yeu à cette date (Fig. 2), on constate qu'une première baisse de pression a eu lieu aux premières heures $d u$ samedi 13 mars 1937, suivie par une deuxième baisse de pression correspondant à ce que voit la simulation de la ré-analyse NOAA. Le météorologue met ainsi en évidence quatre centres dépressionnaires actifs pour cette tempête (Fig. 5), dont il suit chacune des trajectoires et pointe les caractéristiques propres.

Les 147 tempêtes survenues depuis 1979 sont faciles à documenter, grâce aux ré-analyses ERA Intérim, et à verser dans une base de données VIMERS. Les tempêtes plus anciennes peuvent aussi l'être en combinant expertise météo, ré-analyses et archives. Les tempêtes de 1896, 1904, 1924 et 1937 ont déjà été étudiées. 

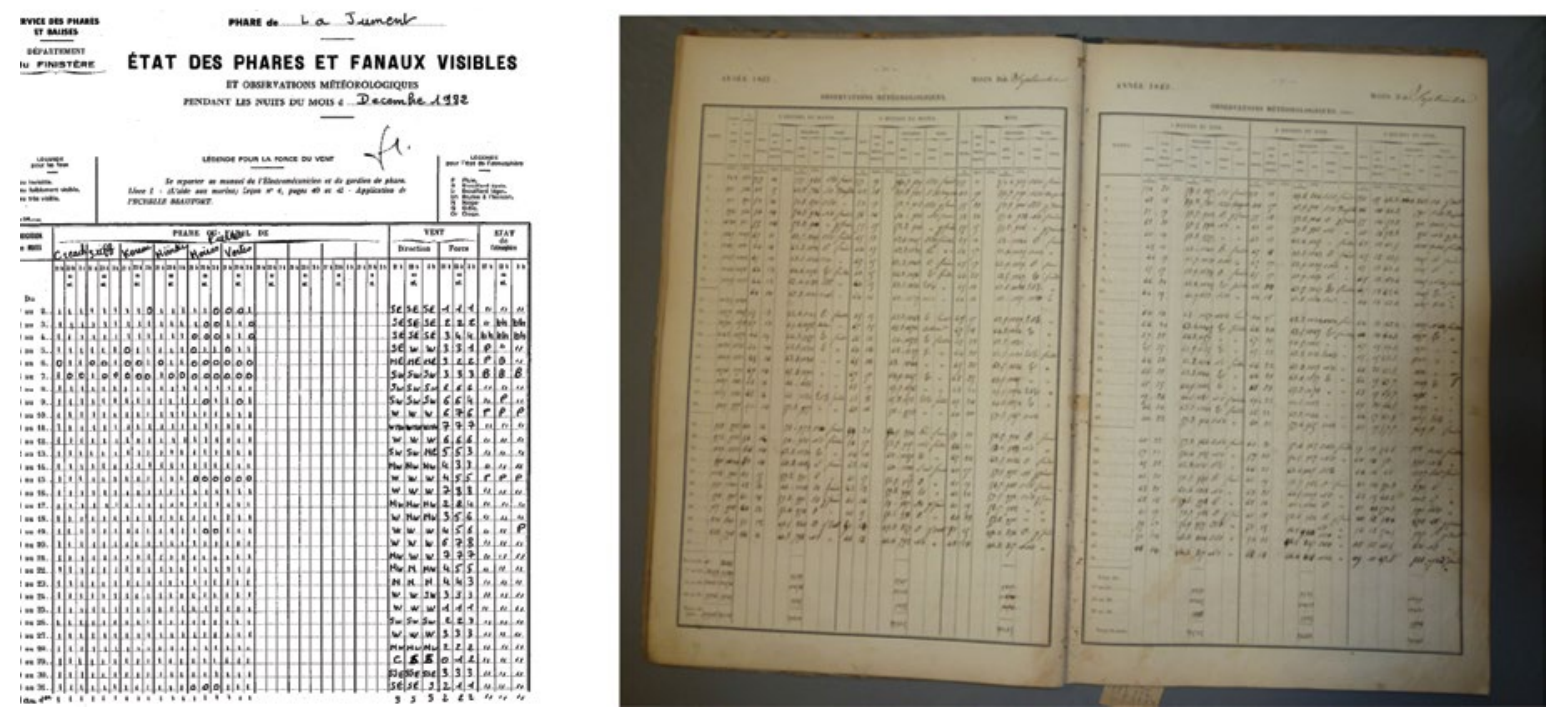

Fig. 4 : A gauche, des informations recueillies par le service des Phares et Balises (Cetmef) et à droite, un recueil de données météorologiques rattachées à un marégraphe (Shom).
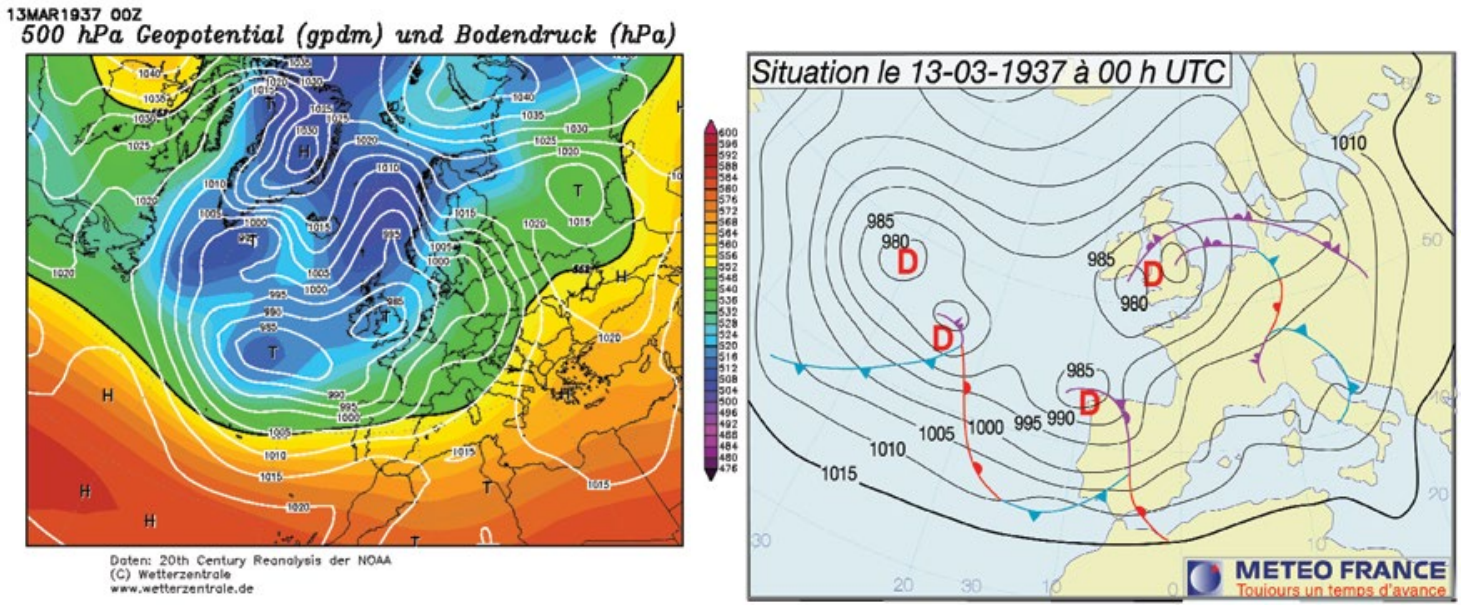

Fig. 5 : A gauche, la simulation de ré-analyse pour le 13 mars 1937 (NOAA, ré-analyses du XX'me siècle) et à droite, la restitution de l'expertise du centre météorologique de Nantes (Météo-France).

\section{LES PIRES TEMPETES PLAUSIBLES}

\section{III.1. Typologie des tempêtes et recherche d'analogues}

La documentation compilée ne suffit pas à désigner les pires tempêtes possibles, ni à dégager des évènements représentatifs : il faut classer les tempêtes sur des critères mathématiques, en établissant des variables rendant compte de leurs propriétés météorologiques. Ainsi, pour chacune des 147 dates ultérieures à 1979 présentant un caractère tempétueux, les experts de Météo-France ont déterminé la trajectoire du centre actif de la tempête au pas de temps tri-horaire, le module et la direction de la vitesse de déplacement, la pression-mer au centre, la pression-mer minimale sur le territoire breton, la vitesse moyenne des vents sur les façades maritimes, la durée totale de la tempête, la distance entre le centre actif et la pointe Finistère, etc.

Sept tests ont été réalisés pour alimenter une démarche de classification reposant sur une classification hiérarchique ascendante (dissimilarité mesurée par la distance euclidienne et le critère de Ward), et sur une méthode k-means. Cette démarche, pratiquée par Météo-France pour déterminer les types de temps, reste sous la maîtrise de l'expert qui veille à ce que les classes statistiques aient un sens météorologique.

De ces sept tests, on conserve finalement celui (Fig. 6) qui fait reposer la classification sur les seules variables suivantes : pression minimale du centre d'action, positions géographiques des points extrêmes de la trajectoire, et composantes du vecteur déplacement moyen.

On en tire une classification en sept familles à la fois stables et consistantes (Fig. 7), présentant chacune des combinaisons de caractéristiques propres (notamment, la pression minimale au centre en $\mathrm{hPa}$ et la vitesse de déplacement en km/h, Fig. 8).

Grâce à cette typologie, on établit une analogie entre les tempêtes anciennes (pour lesquelles les données sont trop fragmentaires pour alimenter une simulation numérique) et les tempêtes plus récentes (qu'il est possible de simuler avec 


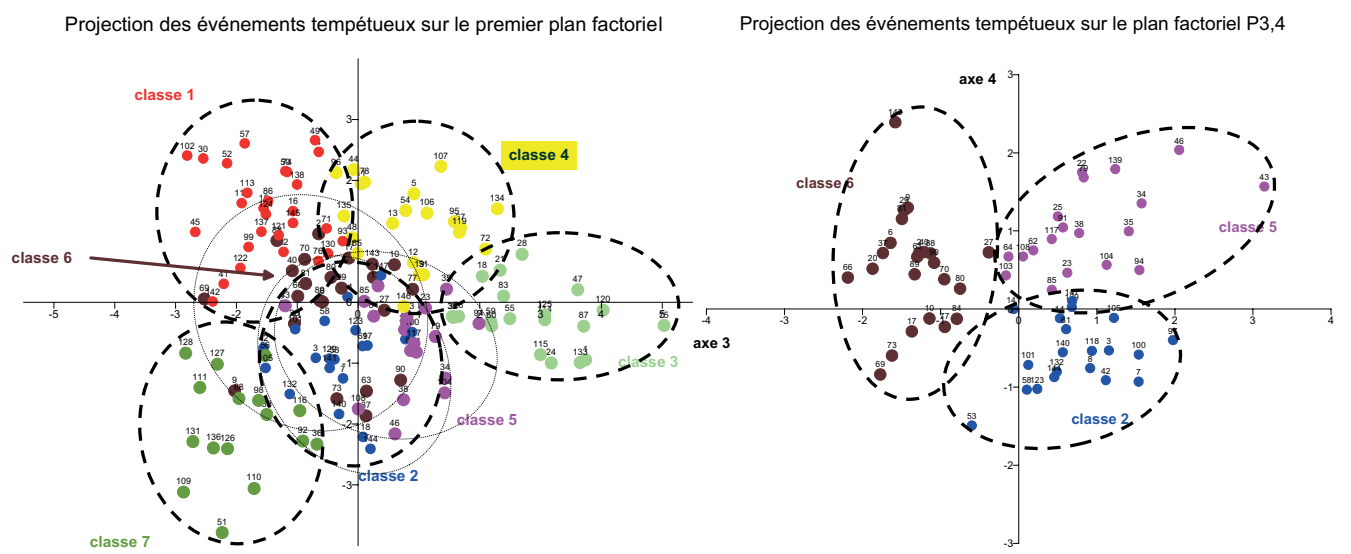

Fig. 6 : Projection des évènements tempétueux sur le premier plan factoriel (à gauche) et sur le plan P3,4 (à droite) (Météo-France).
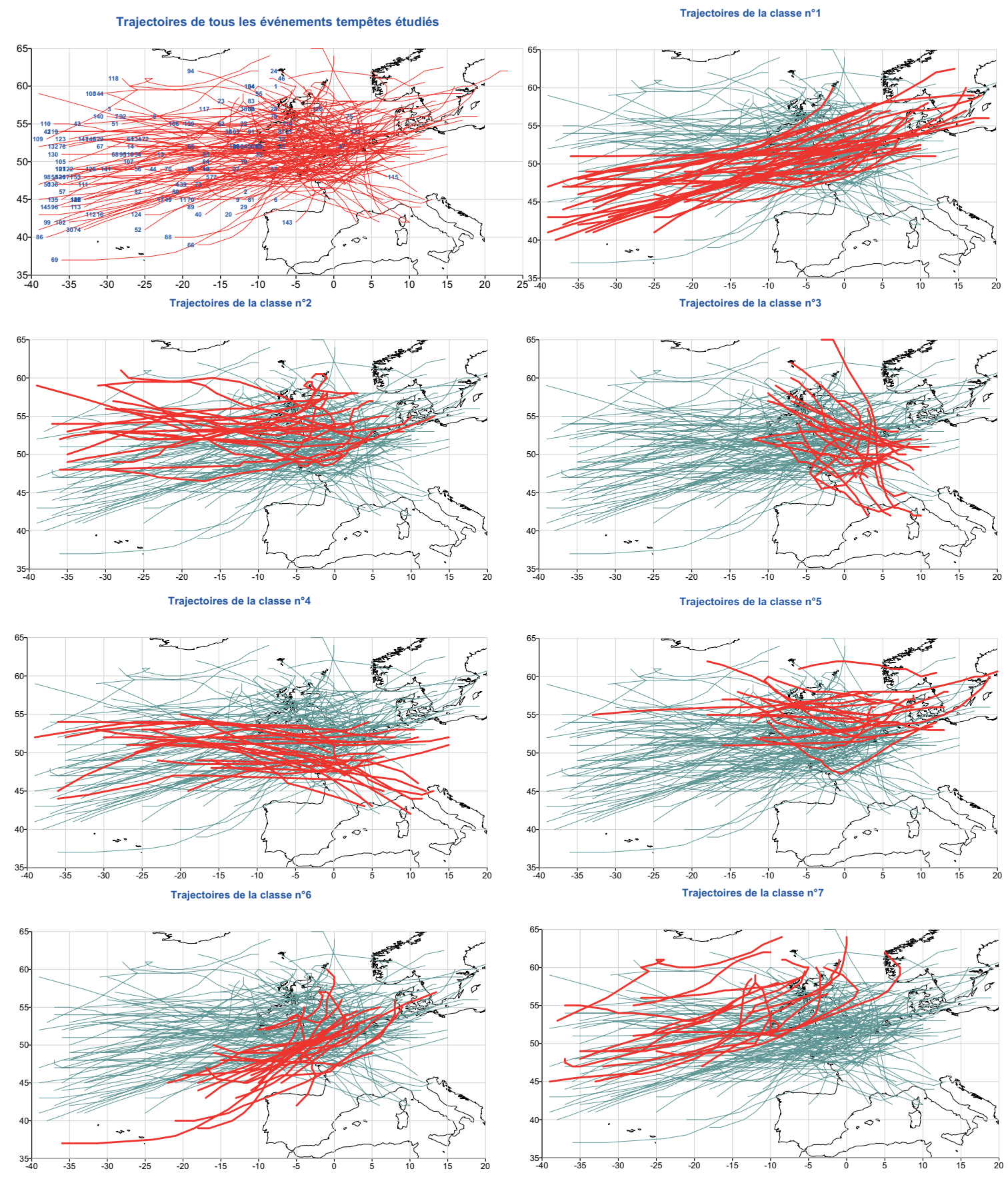

Fig. 7 : Trajectoires des 147 tempêtes et répartition dans les sept familles issues de la classification (28 tempêtes en classe 1, 20 en classe 2, 20 en classe 3, 21 en classe 4, 19 en classe 5, 23 en classe 6, 16 en classe 7) (Météo-France). 

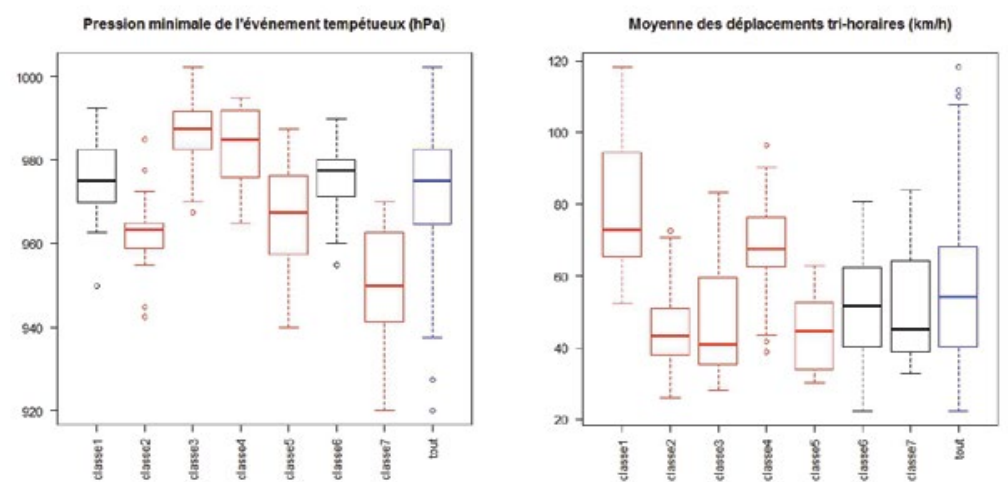

Fig. 8 : Distribution des tempêtes de chaque famille selon les variables de synthèse (Météo-France).

le modèle Arpège de Météo-France). L'exercice n'est toutefois pas déterministe, et il subsiste couramment une hésitation entre plusieurs tempêtes analogues d'une même tempête historique selon qu'on privilégie l'analogie sur les paramètres météorologiques, ou sur les paramètres géographiques.

\section{III.2. Les pires tempêtes plausibles dans le filet de la prévision d'ensemble}

Les pires tempêtes fictives les plus plausibles sont les tempêtes auxquelles on a échappé de peu sans le savoir : à partir d'une situation donnée, le système météorologique peut évoluer de plusieurs manières différentes, et la tempête réelle n'est qu'une seule de ces manières, dont la réalisation a effacé toutes les tempêtes alternatives que la situation initiale rendait possibles.

La Prévision d'Ensemble du modèle Arpège (PEARP) dévoile en partie ces tempêtes alternatives : on calcule, pour une situation météorologique donnée, une fluctuation raisonnable des paramètres du modèle Arpège qui influencent le plus l'incertitude d'évolution du système, et on lance 35 simulations propageant aux mêmes horizons temporels les conséquences de ces fluctuations. La situation historique fait souvent partie de ces 35 simulations, mais chacune a ex ante les mêmes chances de survenance que les 34 autres. L'analyse de la PEARP permet de regrouper les membres qui présentent des évolutions semblables, afin d'établir une densité de probabilité de ces évolutions, et notamment, de prédire les événements extrêmes en les assortissant d'une probabilité. Il est difficile d'apprécier la pertinence de ces prédictions d'événements rares en raison justement de leur rareté, mais il est avéré que, (i) en rejouant des situations extrêmes passées, la prévision d'ensemble peut comporter parmi les membres simulés au moins un membre restituant l'extrême (Marsigli, 2006), et que (ii) les probabilités de prévision d'événements extrêmes sont meilleures pour la variable "vent " que pour d'autres variables (la variable «précipitation », par exemple) (Petroliagis, 1997).

Dans VIMERS, la prévision d'ensemble nous permet d'identifier, à partir d'une date précédant une tempête historique, les membres présentant une situation pire que la situation réalisée : on aurait alors une tempête fictive à la fois pire que la tempête historique, et plausible, puisque ayant ex ante une probabilité d'occurrence comparable (dans un rapport maximal de 1 à 35 ) à celle de la tempête finalement survenue.

Par exemple, la tempête Joachim (16 décembre 2011) appartient à la classe $n^{\circ} 1$. La PEARP (Fig. 9) fournit 35 membres différents à partir de la situation du 14 décembre 2011 (18 h). Le $13^{\text {ème }}$ membre est le plus proche de la situation effectivement réalisée le 16 décembre 2011, avec $980 \mathrm{hPa}$ au centre ; mais les $17^{\text {ème }}$ et $23^{\text {ème }}$ membres ont une pression-mer au centre descendant à $960 \mathrm{hPa}$
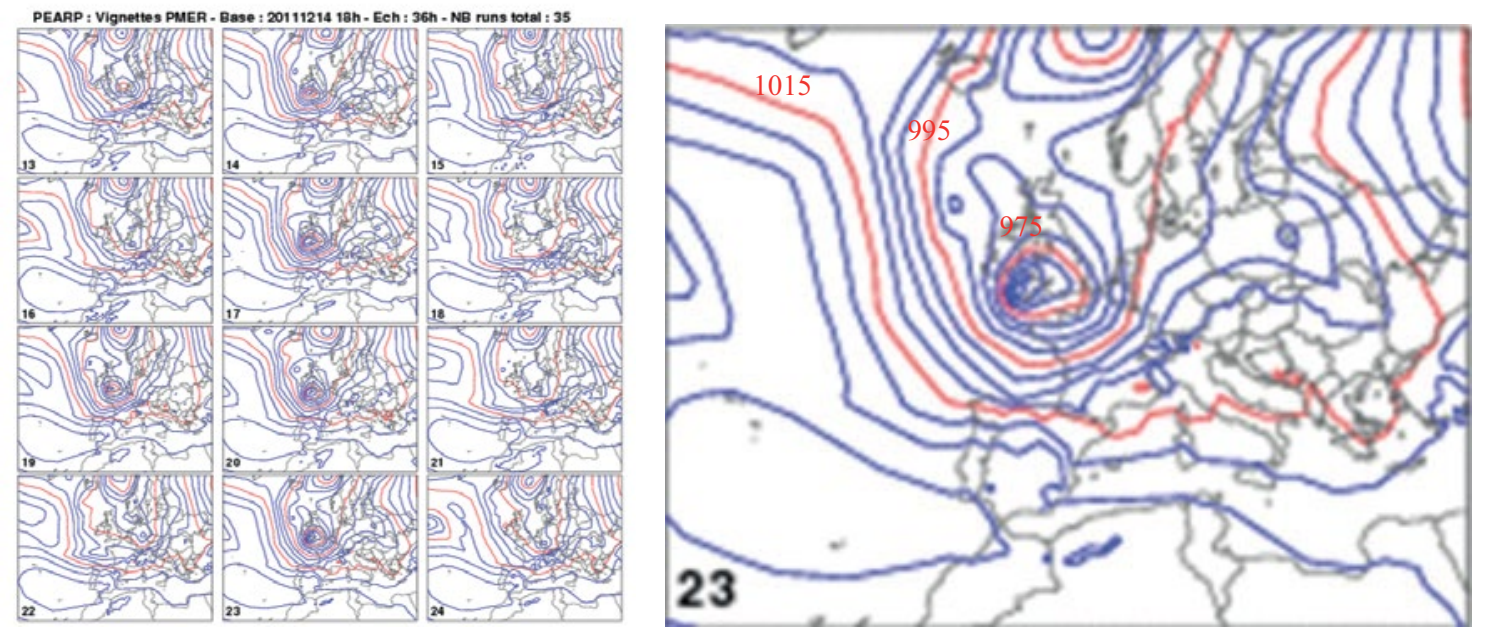

Fig. 9 : Membres $n^{\circ} 13-24$ de la PEARP à 36 h basée sur la situation du 14/12/2011 (18h), zoom sur le membre $n^{\circ} 23$ (Météo-France). 
environ : elles peuvent être analysées comme les 147 tempêtes historiques, constituer le forçage atmosphérique des modélisations hydrodynamiques et majorer la tempête Joachim tout en demeurant dans les limites de la plausibilité réaliste.

La même analyse PEARP menée sur la situation de la tempête Xynthia (27-28 février 2010), qui appartient à la classe $n^{\circ} 6$, ne met en évidence aucun membre dont le centre actif serait plus creusé que la situation historique. Imaginons qu'on ne trouve aucun membre PEARP pire que les plus fortes tempêtes historiques de cette classe $\mathrm{n}^{\circ} 6$ : se dessinerait alors l'hypothèse que les tempêtes historiques constituent, pour cette classe, un maximum relatif dans le périmètre de plausibilité fixé. Toutefois, la robustesse de cette hypothèse serait alors à confronter à une analyse similaire d'autres systèmes de prévision d'ensemble. Ainsi, l'archive de la prévision d'ensemble du modèle européen (EPS) montre, parmi les 50 membres initialisés au 27/02/2010 (minuit) pour l'échéance de $24 \mathrm{~h}$, un (seul) membre (numéro 19) présentant un minimum dépressionnaire (environ $960 \mathrm{hPa}$ ) inférieur à celui (environ $970 \mathrm{hPa}$ ) de la situation réelle pour une latitude similaire.

\section{CONCLUSION}

Le bilan du programme VIMERS à mi-parcours est encourageant : la base de données VIMERS est déjà riche d'évènements récents et anciens, et 25 tempêtes de référence viennent d'être simulées dans Arpège. Plusieurs tempêtes ont été rejouées dans la prévision d'ensemble et les pires membres sont en cours de traitement. Ces conditions atmosphériques vont ensuite forcer les modèles hydrodynamiques de Météo-France et du Cetmef, en concomitance avec des marées défavorables établies par le Shom.

Plusieurs difficultés méthodologiques restent cependant à surmonter.

Comment, par exemple, intégrer ces entités virtuelles non probabilisées dans une évaluation (probabiliste) des risques ? Comment conserver une information de la probabilité d'occurrence ex ante des événements historiques qui les maintiennent dans un même plan de comparaison avec les autres événements possibles pour la même échéance, alors que, ex post, l'événement réalisé se voit nécessairement attribuer une probabilité de $100 \%$ et les événements fictifs concurrents possibles une probabilité de $0 \%$. Ou encore, avant d'intégrer des tempêtes fictives dans les échantillons historiques pour enrichir l'analyse des situations extrêmes, faut-il s'assurer qu'elles demeurent dans la même classe typologique que la situation historique de référence à laquelle on les compare? Des discussions sont engagées avec l'IRSN et EDF-R\&D pour adapter les approches statistiques.

De même, comment combiner ces événements tempétueux avec les conditions de marée pour maximiser, de manière réaliste, la conjonction qui constitue le risque côtier ? Faut-il combiner chaque tempête, historique ou fictive, avec les conditions de marée historiques, avec ces conditions décalées de quelques heures ou avec une marée extrême ? La plausibilité de ces conjonctions doit être suffisante pour être acceptée par les gestionnaires du risque littoral, tout en mettant réellement à l'épreuve les plans élaborés par eux.

La directive Inondations, qui exige l'examen d'un évènement extrême, encourage les autorités publiques à explorer cette voie pour déboucher sur des usages opérationnels. Ces usages sont par exemple : l'information préventive sur les risques littoraux, les scénarios de "shock-tests " auxquels pourraient être soumis des installations sensibles sur le littoral, ou d'exercices de gestion de crise sur des évènements inédits. Météo-France, en accord avec ses partenaires et ses co-financeurs, met à la disposition de la communauté scientifique l'ensemble des matériels produits durant ce programme d'études. Des informations vulgarisées seront également mises en ligne sur l'Internet à destination du grand public.

\section{REMERCIEMENTS}

Les auteurs remercient F. Richard et D. Donnart, de la DREAL Bretagne, S. Pennanguer et J. Paugam, de la Région Bretagne, ainsi que C.-M. Duluc, L. Bardet et Y. Hamdi, de l'IRSN, C. Perherin, A.-L. Tibéri-Wadier, F. Bouttes et X. Kergadallan, du Cetmef, C. Daubord et G. André, du Shom, M. Benoit, EDF-R\&D, et bien sûr, T. Madec, H. Le Cam, P. Germain, G. Simon, M. Aïdonidis, J.-L. Lepape, B. Dubois, H. Canu, I. Laurent, R. Maupaix, B. Saliou, L. Porhel, R. Millour, C. La Verge, F. Rabier, P. Arbogast et D. Paradis, de Météo-France, pour leur concours précieux aux réflexions scientifiques et techniques.

\section{REFERENCES}

Baraer F., Le Cam H., Madec T. (2013) - Rapport d'étude VIMERS. Météo-France, mars 2013

GARNIER E. (2012) — Histoire des tempêtes. Fédération Française des Sociétés d'Assurance. Les cahiers de l'assurance. 91

Marsigli C., Montani A., Nerozzi F., Paccagnella T., Tibaldi S., Molteni F. And Buizza R. (2001) - A strategy for highresolution ensemble prediction. II: Limited-area experiments in four Alpine flood events. Q.J.R. Meteorol. Soc. 127 : 2095-2115

Petroliagis T., Buizza R., Lanzinger A. And Palmer T. N. (1997) - Potential use of the ECMWF Ensemble Prediction System in cases of extreme weather events. Meteorol. App. $4: 69-84$

Pouvreau N. (2008) - Trois cents ans de mesures marégraphiques en France: outils, méthodes et tendances des composantes du niveau de la mer au port de Brest. Thèse. Université de La Rochelle. $466 \mathrm{p}$

Riviere G., Arbogast P., Maynard K. And Joly A. (2010) - The essential ingredients leading to the explosive growth stage of the European wind storm Lothar of Christmas 1999. Q.J.R. Meteorol. Soc. 136 : 638652

SChoenEnWALd N. (2013) - Les tempêtes en France et dans les îles britanniques: des aléas aux évènements. Thèse de doctorat en géographie soutenue le 5 mars 2013, Université Paris-I Panthéon-Sorbonne 